\title{
Point-of-Care Testing in the Time of P5 Medicine: A Preface
}

\author{
Vivek Shetty* and Masaki Yamaguchi ${ }^{1}$ \\ Section of Oral \& Maxillofacial Surgery, 23-009 UCLA Health Sciences Center, \\ Los Angeles, CA 90095-1668, USA \\ ${ }^{1}$ Department of Mechanical Engineering \& Robotics, Graduate School of Science \& Technology, \\ Shinshu University, 3-15-1 Tokida, Ueda, Nagano 386-8567, Japan
}

(Received February 1, 2016; accepted April 28, 2016)

Keywords: P5 medicine, point-of-care, biosensors, connected health

The expectations of point-of-care technologies (POCT) are being increasingly shaped by the patient-centric narrative of P5 (predictive, preventive, personalized, participatory, and populationbased) medicine. Shifting the focus of our healthcare system to a personalized and preemptive approach will depend partly on the availability of field-deployable measurement technologies that provide rapid biometric information to providers and patients. In order to respond to the changing healthcare paradigm and hasten the integration of emerging sensor materials into clinically useful point-of-care (POC) approaches, it is essential that the sensor materials community understand and appreciate the clinical context, expectations, and aspirations of the healthcare consumers. After reviewing the drivers of the P5 medicine initiative, we review the context within which the POCT will be used, and some of the challenges associated with delivering portable, fast response, and highly reliable measurement technologies that can be used with complex biofluids in a range of settings by a variety of users. We conclude by emphasizing the potential synergies of linking POCT's to the "connected health" model which integrates mobile technologies, low-cost hardware, healthcare IT systems, big data analytics, and the Internet of Things (IoT) to connect all stakeholders for a timely sharing of pertinent health information necessary for timely and personalized healthcare.

\section{Introduction}

Healthcare throughout the world is in the midst of dramatic change. Social, economic, technological, and political forces are radically reshaping how and where healthcare is delivered and how it is paid for. The drivers are many: aging populations with more chronic conditions, a reengineering of the focus of health care systems from caring for acutely sick individuals to managing the health of entire populations, growing health-provider shortages, and a "downshifting" of care delivery across the provider spectrum (i.e., from specialists to generalists to physician aides to healthcare workers), a replacement of the traditional fee-for-service reimbursement model (wherein providers are paid for each service rendered to a patient) by capitation models (in which providers are paid a "lump sum" per patient regardless of how many services the patient receives), and an eager adoption of the burgeoning innovations in health technologies, digital health tools, and big data.

"Corresponding author: e-mail: vshetty@ucla.edu 
The challenges of providing quality and affordable healthcare widely within this context are intensified by the growing push to transform healthcare from its current one-size-fits-all paradigm that is based on "standards of care", to an approach tailored to each individual.(1) Over time, the integrative, systems-approach of $\mathrm{P} 4$ (predictive, preventive, personalized, and participatory) medicine espoused by Leroy Hood (2013) has been expanded to embrace a population-health perspective, and use of the term "P5 medicine". ${ }^{(2,3)}$ Fueled by the "omics" breakthroughs that now allow detailed biological profiling of individuals, and by the scientific advances of the past decade, P5 medicine seeks to individualize healthcare to the patient, allowing providers to match therapies to patients based on their biological profiles, to proactively identify an individual's susceptibility to health conditions, to detect early disease and preempt disease progression, and to anticipate the patient's response to a particular therapy. The P5 approach shifts the emphasis in medicine from reaction to prevention and from disease to wellness. Concurrently, the bottom-up strategy is expected to also increase the efficiency of the healthcare system by improving quality, accessibility, and affordability. ${ }^{(4)}$ P5 medicine strives to empower individuals with timely access to understandable health information, allowing them to take more responsibility for their well-being and health behaviors, become better educated about their diseases, and ultimately more engaged collaborators with their health providers.

\section{The Case for Point-of-Care Technologies (POCT)}

Realizing the promise of P5 medicine depends significantly on the development of measurement technologies and analytical approaches for detailed biological profiling of individuals. Of particular interest are point-of-care (POC) devices that do not involve an elaborate laboratory infrastructure or specialty expertise to provide health data to end-users. Requiring minimal preparation of biofluids, ranging from blood to saliva and urine, and analytical targets that include proteins, metabolites, and nucleic acids, POCT endeavor to deliver speedy and actionable information to healthcare providers or to patients themselves. ${ }^{(5)}$ Advantages inherent to POC devices include portability, ease of operation, accuracy, and reliability of results. However, the most compelling attribute of POCT is a shortened turnaround time (TAT), namely, the time from initiation of the test order to the implementation of the clinical decision. ${ }^{(6)}$ Self-administered at the patient's home or conducted near the patient in the hospital or clinic, POC testing provides an "answer" on the spot. Existing in-home POC systems, such as glucometers, are already decreasing costs and increasing patient convenience by reducing the frequency of hospital visits, associated travel expenses, and lost worktime. (7) Self-monitoring also gives greater responsibility to the patient, enhances their understanding of their medical condition, and improves patient adherence to prescribed treatments. Performed in clinical settings with TAT's $<15$ min (the typical duration of a clinical encounter), POC tests allow the healthcare provider to promptly initiate definitive medical treatment instead of starting off with empiric therapy and subsequently refining it after the laboratory results become available in the days or weeks after the initial encounter. Also, on-site POC testing is a leaner process that significantly diminishes the processing errors and expenses linked with specimen collection, transport, processing, analysis, and reporting steps associated with centralized laboratory tests.

An illustrative example is human immunodeficiency virus (HIV) infection; 38.9 million people worldwide are infected with HIV, but only $10 \%$ are aware of their status. ${ }^{(8)}$ HIV testing, the foremost imperative for preventing further transmission and to caring for those infected, is 
traditionally performed outside of the clinical encounter in centralized laboratories. ${ }^{(9)}$ Tested individuals are asked to return several weeks post-test to learn the results, and for treatment and prevention counseling, to reduce risky behaviors among those found to be infected. However, more than one third of those tested do not return for results, representing a missed prevention opportunity. ${ }^{(10)}$ The impact of the TAT lag is significantly worse in low resource settings, or regions with a very high prevalence of HIV and lacking in adequate healthcare infrastructure and specialist personnel. POC testing for screening, diagnosis, treatment initiation, and the monitoring of HIVtreatment efficacy helps resolve these deficiencies by allowing infected individuals to learn their serostatus quickly and by connecting them to medical care. ${ }^{(9)}$ Low cost, user-friendly, and accurate HIV diagnostic tests, patterned after home pregnancy tests, are particularly enabling for those individuals who may not otherwise test. Direct ways of measuring the amount of the HIV virus present (viral load) allows monitoring of how advanced the infection is and how well treatment is working. ${ }^{(1)}$ The timely initiation of POC-facilitated antiretroviral therapy, especially in the case of pregnant women, greatly helps reduce the risk of further transmission and markedly improves survival by ensuring successful viral suppression. ${ }^{(12)}$

Or consider the case of psychiatric disorders following the experience of a traumatic injury. Significant subsets of injury survivors $(\sim 30 \%)$ go on to develop debilitating psychiatric sequelae such as post-traumatic stress disorder (PTSD) and major depressive disorder (MDD) that are debilitating, and difficult to treat once established. ${ }^{(13)}$ Early identification of maladaptive stress reactions provides the opportunity for timely mental health interventions that could prevent the crystallization of maladaptive stress reactions into entrenched psychiatric sequelae. ${ }^{(13)}$ However, due to the syndromic (symptom based) nature of psychological assessments, front-line providers have difficulty differentiating transient reactional distress from the prodromal stages of more serious mental health illnesses. Psychological assessments are also very time and resource intensive, have numerous reliability and validity issues, and are burdened by dispositional and reporting biases. ${ }^{(14,15)}$ One approach has been to exploit the fluctuating levels of peripherally-expressed biomarkers of the neuroendocrine stress response to a trauma to quantify the nature and magnitude of the psychological impact. Research groups have been developing portable biosensors to allow for near "real-time" detection and quantification of stress-response biomarkers, including salivary $\alpha$-amylase and cortisol. ${ }^{(16-19)}$ Executable algorithms and treatment decision trees inputted into the biosensor microprocessor would eventually be able to analyze patterns in salivary stress biomarkers to generate simple feedback that is readily accessible to even a non-specialist. Enabled by this "smart systems" technology, trauma care providers would be able to identify "at-risk" patients, conduct timely restorative interventions, or refer individuals for specialized mental health care, in addition to monitoring progress and response to therapy. ${ }^{(19)}$

\section{Biosensors and Liquid Biopsies}

The utility of POC biosensors derives largely from their ability to extract biometric information about health and disease states using informative biological indicators. Traditional biopsies require invasive sampling of primary tissue to evaluate the state of tissues and organs. This approach can be challenging or even unfeasible in hard-to-reach tissues, including brain or cardiac tissue. ${ }^{(20)}$ In contrast, the "liquid biopsies" performed by POC biosensors take advantage of tissue proxies such as proteins and protein fragments that are released by tissue cells into body fluids. ${ }^{(21)}$ The 
information contained within the peripheral biofluids are then used to determine a disease trait (risk factor or risk marker), disease state (preclinical or clinical), or disease rate (progression). (22) Beyond the target analytes (e.g., secreted proteins, sugars, cells, nucleic acids, microbes), the development of biosensors is also driven by the typical setting for their use (i.e., acute or primary care setting, home care, low resource setting, or disaster setting) and the intended end-user (patient of the healthcare provider). The functionality of the biosensor will also vary according to whether the POC device is going to be used to monitor exposure to environmental toxins (biomonitoring) or to indicate disease by helping to identify disease (diagnostic), predicting the likely course of the disease in untreated individuals (prognostic), identifying patients who are more likely to respond to a given therapy (predictive), or monitoring disease progression or effects of interventions (monitoring). ${ }^{(23)}$

An early decision facing the POC technologist is the choice of biofluid to source the biomarkers: blood, saliva, urine, or sweat. Each biofluid offers different opportunities in terms of biomarker composition and availability, ease of sample collection, and processing. Though attractive due to its very high protein concentration, blood presents considerable analytic challenges due to the extremely wide dynamic range (12 orders of magnitude) of its constituent proteins and very low abundance of potentially informative biomarkers. ${ }^{(24)}$ Urine samples demonstrate a high degree of variability in volume, $\mathrm{pH}$, and urine components due to age, health, diet, and proteolysis while the urine is stored in the bladder. In our own studies, we use saliva as the biofluid of choice within the context of stress biomarker measurements. ${ }^{(25)}$ Containing many biomarkers linked to stress disorders, cancer, cardiovascular, metabolic, and infectious diseases, saliva manifests a broad spectrum of normal and disease states. ${ }^{26,27)}$ Saliva's potential utility was underscored by Alterovitz et al. (2008) who, using an information theory framework to conceptualize tissuebiofluid interactions as information channels, concluded that saliva is one of the most informative biofluids when it comes to biomarker discovery and measurement. ${ }^{(28)}$ Beyond the biomarkers it contains, saliva offers many practical advantages. For patients, supplying a saliva sample does not evoke the anxiety associated with drawing a blood sample, and causes less embarrassment than producing a urine specimen. Saliva samples can be obtained without difficulty from children and individuals with physical or mental handicaps. ${ }^{(19,29)}$ Saliva does not clot and is neither infectious nor considered biohazardous, making processing and disposal much easier. Furthermore, multiple saliva samples can be collected over a period of time without the use of specialized equipment or personnel, thereby allowing for easier monitoring of time-dependent changes in biomarker levels for extended periods. Saliva is particularly pertinent to the study of stress-related disorders because the anxiety and pain associated with a venipuncture can distort the profiles of the stress biomarkers. Overall, saliva provides a compelling basis for inexpensive, noninvasive, and easy-to-use diagnostic technology.

\section{A Technical Brief for POC Biosensors}

All too often, POC biosensing technologies fail to advance beyond the research setting to gain acceptance by the broader clinical community and make a meaningful impact on health care. A major flaw is the excessive focus on innovative materials and technologies as a springboard, instead of real-world applications informed by end-user insights. Using the intended clinical application and measurement target as a point of departure, biosensor development should build on the ASSURED criteria articulated by the World Health Organization for POC diagnostics: 
affordable, sensitive, specific, user-friendly, rapid and robust, equipment-free and deliverable to end-users. ${ }^{(30)}$ The biosensor should be capable of measuring the target analyte/s in small volumes of complex biofluids such as saliva or blood, with acceptable sensitivity, selectivity, and reliability. ${ }^{(5)}$ Cost is a major consideration, especially in low resource settings. ${ }^{(31)}$ One strategy for reducing cost is to create durable multi-use readers capable of conducting different tests, and to couple them with inexpensive, disposable POC chips or strips that incorporate a miniaturized sample preparation, separation, and detection system. Smartphones enabled for clinical microscopy or colorimetric readouts have great potential as low-cost readers. ${ }^{(32)}$ Increasingly, assay platforms use inexpensive substrates such as bioactive paper on which the functional recognition biomolecules are physically immobilized on cellulosic supports. ${ }^{(5)}$ Additionally, selective recognition and reliability of the POC system can be advanced through the use of synthetically-generated aptamers instead of conventional antibodies that can vary from batch to batch and vendor to vendor. ${ }^{(5)}$ Label-free detection systems with their simplified assay steps and lower complexity and costs are another attractive option. ${ }^{(33)}$ Broader adoption of the high throughput and scalable manufacturing techniques developed by the microelectronics industry will accelerate the large-scale manufacturing of miniaturized, integrated POC devices that can be deployed cost-effectively in a variety of settings.

The expectation of rapid and reliable test results in a variety of settings greatly increases the importance of error-free testing and test result reliability. In contrast to the structured, controlled testing of centralized laboratories where any errors originate mostly in the pre- and post-analytic phases of testing, the errors involved with POC testing occur primarily in the analytic phase. Hence, the measurement capabilities of POC devices must compensate for a large variation in the use settings and conditions, and accommodate limited understanding or no training on the part of the user. ${ }^{(31)}$ As with laboratory based analyzers, a stringent quality control element is an essential requirement of POC biosensors. Beyond simple battery and display checks, the biosensor needs to have self-check capabilities with inbuilt calibrators that ensure and maintain good control and reliability of the system. Given that the focus of a POC system is to provide clear and understandable health information, the motor, sensor, and cognitive needs of the end-users should be placed at the center of the design process. ${ }^{(34)}$ Utilizing a human-centered design approach, the development and evaluation of the POC interfaces should take a "black box" approach wherein the technical complexity of the assay process is effectively hidden from the end-user.

Until recently, most POC tests focused on single biomarkers, and the assay requirements were relatively simple. However, the explosion of knowledge in genomics, transcriptomics, proteomics, and metabolomics has led to an increasing recognition that single biomarkers cannot adequately capture the complexity of the dysregulations that lead to disease. Instead, a systems biology approach that puts an emphasis on multimarker fingerprints is better positioned to describe the disease process in question while improving the sensitivity, specificity, and predictive performance of individual markers. ${ }^{(35,36)}$ However, accommodating the need for parallel measurements of multiple analytes greatly increases the technical challenges involved in assay platform development. Simultaneous detection of multiple biomolecules (multiplexing) is often complicated by issues such as the matrix effects of complex biofuids, non-specific binding, and cross-reactivity due to the binding of capture antibodies to multiple targets or vice versa. Solutions have included the selection of high affinity ligands for target biomarkers, and exploiting the unique properties of nanomaterials such as graphene, carbon nanotubes or quantum dots to develop sensing systems for multiplexed detection. ${ }^{(5)}$ Nano-enabled biosensors that utilize nanotechnology for recognition, transduction, or 
both, offer much potential with their inherent high surface-to-volume ratios, multi-functionality, and exceptional physicochemical properties. ${ }^{(37)}$ The individual elements of high density arrays can be functionalized with different capture ligands, thus allowing for concurrent measurements of positive/negative controls and analytes of varying concentrations while controlling for signal fluctuations and enhancing sensitivity and specificity.

\section{Biosensors and Connected Health}

At its very core, P5 medicine is a participatory approach to care delivery and seeks to adapt the healthcare system to the patient rather than the other way around. Thus, the integration of POC devices within the e-health ecosystem should be an essential design consideration. The emerging "connected health" model of healthcare leverages the convergence of mobile technologies, lowcost hardware, healthcare IT systems, big data analytics, and the Internet of Things (IoT) to connect all stakeholders through a timely sharing of accurate and pertinent information regarding a patient's health status. ${ }^{(38)}$ Using the ubiquitous smartphone as a local data acquisition node, sensed information from biosensors can be communicated securely to private data vaults for merging with electronic medical records, integration with other health data streams, big data analytics and eventually, timely feedback to both provider and patient. Such connected POC devices with a feedback loop would be an incredibly valuable platform for here-and-now health information, providing patients with a meaningful, understandable view of the interconnected aspects of their health, helping to foster healthy behaviors, patient compliance and engagement, and providing the foundation for real-time, personalized care management programs. Furthermore, automated collection of data and broad integration across devices and systems would allow healthcare systems to extend personalized care to a wider population of patients and help create new care-delivery models that are better coordinated and that emphasize patient self-management. Population-level views, facilitated by low-cost POC devices and geospatial mapping, could reveal emerging patterns of illnesses, detect systematic variation in clinical practice, and help evaluate the comparative effectiveness of treatments related to population characteristics not easily explored by clinical trials. The blending of POCT with wireless connectivity will bring a new dimension of precision, efficiency, and scalability to the way health information is collected, transmitted and interpreted, and fundamentally alter the way medicine is practiced. Most importantly, by facilitating a more holistic and personalized medicine that allows patients to be co-managers of their own health and wellness, POC sensors utilized within a P5 framework could help redirect humanity back into healthcare's increasingly outdated model.

\section{Summary}

Advances in POCT are being shaped increasingly by the narrative of P5 medicine. The success of a prospective shift from curative medicine to a value-based, personalized, predictive, and preemptive medicine relies, in large part, on the development of portable devices for near-patient diagnosis and monitoring. When properly integrated into the connected healthcare paradigm, POCT will allow rapid and accurate capture of important biometric data, facilitate timely therapeutic decisions, minimize practice variations, and improve patient engagement, patient satisfaction, and ultimately, patient outcomes. In order to respond to the changing healthcare paradigm and 
accelerate the incorporation of emerging sensor materials into clinically useful POC approaches, it is critical that the sensor materials community understand and appreciate the clinical context, expectations, and aspirations of healthcare consumers.

\section{Acknowledgements}

This work was supported by NIH/National Institute of Dental and Craniofacial Research Grant R01DE022045-01A.

\section{References}

1 M. G. Aspinall and R. G. Hamermesh: Harv. Bus. Rev. 85 (2007) 108.

2 L. Hood: Rambam Maimonides Med. J. 4 (2013) 12.

3 M. J. Khoury, M. Gwinn, R. E. Glasgow, and B. S. Kramer: Am. J. Prev. Med. 42 (2012) 639.

4 B. Keogh: J. Natl. Cancer Inst. 104 (2012) 12.

5 V. Gubala, L. F. Harris, A. J. Ricco, M. X. Tan, and D. E. Williams: Anal. Chem. 84 (2012) 487.

6 B. Dey, J. N. Bharti, and M. Chakraborty: Int. J. Health Sci. Res. 5 (2013) 82.

7 S. F. Clarke and J. R. Foster: Br. J. Biomed. Sci. 69 (2012) 83.

8 Fact Sheet 2015, http://www.unaids.org/en/resources/campaigns/HowAIDSchangedeverything/factsheet (accessed January 31, 2016).

9 L. M. Bogart, D. Howerton, J. Lange, K. Becker, C. M. Setodji, and S. M. Asch: Public Health Rep. 123 (2008) 494.

10 V. A. Moyer: Ann. Intern. Med. 159 (2013) 51.

11 H. Shafiee, E. A. Lidstone, M. Jahangir, F. Inci, E. Hanhauser, and T. J. Henrich: Sci. Rep. 4 (2014) 416.

12 S. D. Reid, S. J. Fidler, and G. S. Cooke: Clin. Epidemiol. 5 (2013) 387.

13 M. L. O’Donnell, M. Creamer, P. Pattison, and C. Atkin: Am. J. Psychiatry 161 (2004) 507.

14 A. J. Marsella, M. J. Friedman, E. T. Gerrity, and R. M. Scurfield: Ethnocultural Aspects of Post Traumatic Stress Disorder: Issues, Research, and Clinical Applications, 1st ed. (American Psychological Association (APA), 1996).

15 E. G. Triffleman and N. Pole: J. Consult. Clin. Psychol. 78 (2010) 490.

16 M. Yamaguchi, Y. Matsuda, S. Sasaki, M. Sasaki, Y. Kadoma, Y. Imai, D. Niwa, and V. Shetty: Biosens. Bioelectron. 41 (2013) 186.

17 M. Yamaguchi and V. Shetty: Distrib. Diagn. Home Healthcare, D2H2 2012 (2012) 1.

18 M. Yamaguchi, S. Yoshikawa, Y. Tahara, D. Niwa, Y. Imai, and V. Shetty: Sens. 2009 IEEE (2009) 343.

19 V. Shetty and M. Yamaguchi: Oral Maxillofac. Surg. Clin. North Am. 22 (2010) 269.

20 M.-L. Wong and J. Licinio: Mol. Psychiatry 10 (2005) 421.

21 E. Crowley, F. Di Nicolantonio, F. Loupakis, and A. Bardelli: Nat. Rev. Clin. Oncol. 10 (2013) 472.

22 R. S. Vasan: Circulation 113 (2006) 2335.

23 R. Mayeux: NeuroRx 1 (2004) 182.

24 T. D. Veenstra, T. P. Conrads, B. L. Hood, A. M. Avellino, R. G. Ellenbogen, and R. S. Morrison: Mol. Cell. Proteomics 4 (2005) 409.

25 T. Robles, V. Shetty, C. Zigler, D. Glover, D. Elashoff, and M. Yamaguchi: Biol. Psychol. 86 (2011) 50.

26 I. D. Mandel: Ann. N. Y. Acad. Sci. 694 (1993) 1.

27 D. T. Wong: J. Am. Dent. Assoc. 137 (2006) 313.

28 G. Alterovitz, M. Xiang, J. Liu, A. Chang, and M. F. Ramoni: Pac. Symp. Biocomput. 13 (2008) 231.

29 Y.-H. Lee and D. T. Wong: Am. J. Dent. 22 (2009) 241.

30 P. Yager, T. Edwards, E. Fu, K. Helton, K. Nelson, M. R. Tam, and B. H. Weigl: Nature 442 (2006) 412.

31 P. Yager, G. J. Domingo, and J. Gerdes: Annu. Rev. Biomed. Eng. 10 (2008) 107.

32 D. N. Breslauer, R. N. Maamari, N. A. Switz, W. A. Lam, and D. A. Fletcher: PloS One 4 (2009) 6320.

33 S. Unser, I. Bruzas, J. He, and L. Sagle: Sensors 15 (2015) 15684.

34 Z. Martina: Human-Centered Design of E-Health Technologies: Concepts, Methods and Applications (IGI Global, 2010).

35 R. Mayeuxs: NeuroRx 1 (2004) 182. 
36 R. S. Vasan: Circulation 113 (2006) 2335.

37 B. He, T. J. Morrow, and C. D. Keating: Curr. Opin. Chem. Biol. 12 (2008) 522.

38 B. M. Caulfield and S. C. Donnelly: QJM Mon. J. Assoc. Physicians 106 (2013) 703. 